\title{
Low-intensity auditory stimulation and the GSR orienting response
}

\author{
ROBERT J. BARRY \\ University of Sydney, Sydney 2006, Australia
}

\begin{abstract}
Sokolov's prediction of enhanced orienting response (OR) magnitude produced by stimulus intensities below about $50 \mathrm{~dB}$ was tested by essentially replicating his within-subjects paradigm with the GSR. Twenty-four subjects were tested with $20,30,40$, and $50 \mathrm{~dB}$ SPL $1,000-\mathrm{Hz} 2-\mathrm{sec}$ tones of minimal significance with a unique order of tone presentation for each subject. A linear relation between GSR magnitude and stimulus intensity was obtained, contrary to Sokolov's prediction. Habituation over eight stimulus cycles was obtained, as required of an OR indicator.
\end{abstract}

Sokolov (1963) indicated that the galvanic skin response (GSR) was studied by Russian workers as a correlate of motor reactions and visual processes from the 1930s, and recognized from extinction studies in the 1950 s as "essentially a manifestation of the OR (orienting response)." His own experiments showed it to be elicited by light, sound, temperature, tactile, proprioceptive and other stimuli of low to moderate intensity, and to habituate rapidly, as required of an OR indicator. The GSR is one of the few measures for which Sokolov has reported actual values. After noting that intensity effects may be masked by ordering effects in stimulus presentation, he provided mean data for 15 subjects of change in resistance averaged over the number of trials required to ob tain complete extinction to all stimuli, as a function of stimulus intensity for $1,000-\mathrm{Hz}$ tones of $2 \mathrm{sec}$ duration. This showed an enhanced effect of near threshold stimuli in eliciting ORs at low intensity, and a linear dependence of OR magnitude on stimulus intensity above this range.

In the West, Hovland and Riesen reported, as early as 1940 , that GSR magnitude was proportional to the intensity of a simple auditory stimulus. Uno and Grings (1965), using white noise from 60 to $100 \mathrm{~dB}$, also found that the magnitude of the GSR was directly related to stimulus intensity and habituated with stimulus repetition. Leavy and Geer (1967) have provided the only attempt to evaluate specifically the enhancement of the GSR reported by Sokolov at low stimulus intensity levels by purporting to replicate the experiment referred to above. Unfortunately, whereas Sokolov used one ordering of intensity per subject (randomized over subjects) and repeatedly administered this cycle of stimuli until habituation occurred, Leavy and Geer repeatedly administered one intensity to each subject for 16 trials. Not surprisingly, they failed to obtain a significant relationship between $O R$ amplitude and stimulus intensity. Raskin, Kotses, and Bever (1969) also used a factorial design in which each subject received

Send requests for reprints to Robert J. Barry, School of Education, University of N. S. W., P. O. Box 1, Kensington 2033, Australia. only one stimulus intensity ranging from 40 to $120 \mathrm{~dB}$. The greater number of subjects in this study (120 compared with Leavy \& Geer's 48), combined with the greater range of intensity, appears to have resulted in the intensity effect reaching significance.

It is worthwhile emphasizing the procedural difference and the issues involved. If a subject is given only one stimulus intensity, Sokolov's neuronal model would predict an initial OR to the first stimulus which would depend on both the stimulus intensity and the past experience of the subject. Rapid habituation would then be expected to occur on subsequent trials since the required model of the stimulus is particularly simple. On the other hand, the situation in which a subject is given repeated cycles of (say) four intensities differs in two respects, the most obvious of which concerns the nature of the hypothesized neuronal model. Since the model is required to cope with a stimulus complex of four stimuli, its setting-up is more difficult and slower, leading to the expectation of larger ORs with slower habituation. Thus, the nature of the OR expected differs between the two experimental paradigms. The second point concerns the benefit of a repeated-measures design in the statistical analysis of the OR. Where one subject provides data at different intensities, his "individual" contribution to each score can be partitioned out (by, for example, a repeated-measures analysis of variance), increasing the power of the analysis. This is important here, since Leavy and Geer reported that the "great intersubject variability with respect to OR strength appeared to be masking possible group differences [p. 105]." Similarly, the remark above regarding Raskin et al's data reaching significance reflects a possible increase in power due to increased sample size.

This difference in expected OR magnitude and habituation rate when between-subject and within-subject paradigms are compared is paralleled in reaction-time (RT) studies. For example, Grice and Hunter (1964) reported that the within-subjects intensity effect (higher intensities producing faster RTs) was much greater than the between-subjects RT-intensity effect. For this reason, it is surprising that Sokolov's use of the within-subject paradigm has not 
been further investigated. This study aimed to rectify that situation with respect to the low-intensity stimuli where Sokolov (1963) has reported enhanced response magnitude.

A final variable to be considered is the so-called "significance" of the stimulus. For example, if a tone regularly precedes a shock, the tone itself will become more significant to the subject, and his response to it will differ from his original response to the undifferentiated tone. Similarly, past experience and, in particular, the experimenters instructions, may result in subjects attributing more or less significance to a given stimulus. Campos and Johnson (1966) varied stimulus significance by verbal instructions requiring different levels of attention by the subject and found a consistent significant effect on the GSR. Kohlenberg (1970) instructed different groups to ignore one particular tone of a cycle of three through several repetitions. This could be expected to focus attention on this tone, increasing its apparent significance over that of the other tones, since it would have to be identified by the subject before he could "ignore" it. As could be expected from this analysis, the ignored tone was found to elicit greater GSRs than the other tones. In this study, an attempt was made to minimize the significance attributed by the subject to the stimulus complex.

\section{METHOD}

Subjects were twenty-four males under 25 years of age who participated to fulfill a requirement of an introductory psychology course. They were seated in the dark in a comfortable armchair in an electrically shielded light-tight sound-attenuated chamber. The GSR was recorded from lead electrodes on the volar surfaces of the first and second fingers of subject's left hand. In addition, three channels of EEG, eye movements, respiration, heart rate, digital pulse volume, and cephalic pulse volume were recorded but are not reported here. Because of the

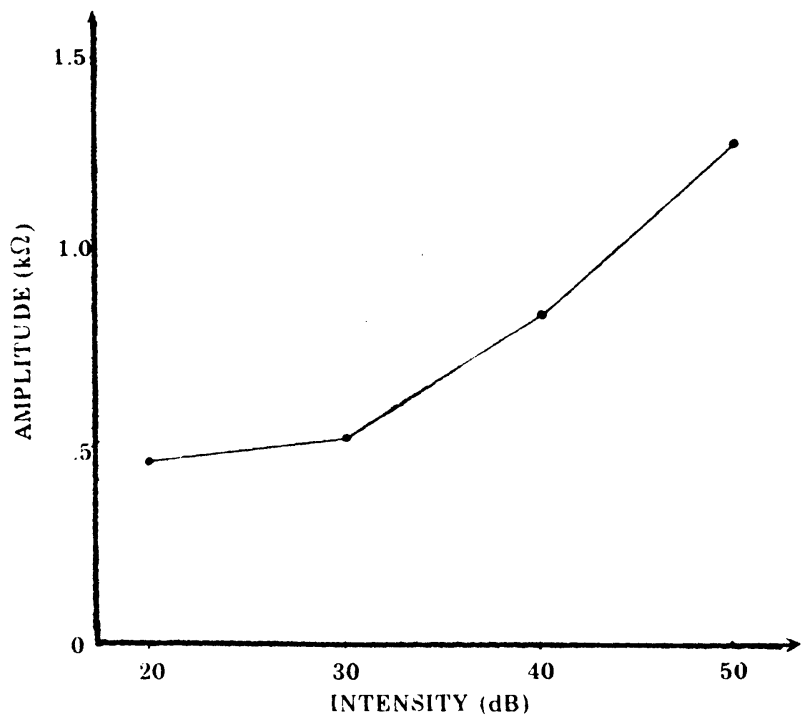

Figure 1. Amplitude of the GSR as a function of stimulus intensity.

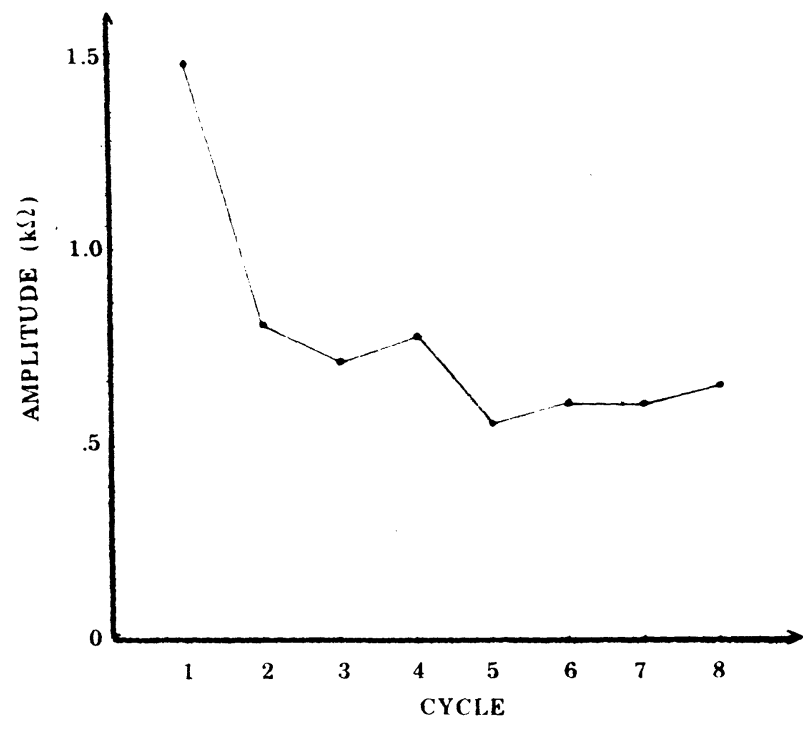

Figure 2. Amplitude of the GSR as a function of repeated cycles of stimulation.

demand on the experimenter's attention imposed by this multiple recording, only ac-coupled resistance changes could be measured, using an E\& M physiograph with a system time constant of $5 \mathrm{sec}$. While this is away from the current trend to the use of conductance change as the measure of the GSR [Lykken \& Venables (1971)], Montague and Coles (1966) have pointed out that the universal validity of the independence of tonic and phasic GSRs (the main reason for moving away from resistance measures) should not be accepted without question. In OR studies. with novel stimuli, it is reasonable to assume rapid changes in both tonic and phasic conductance levels such that a measure reflecting changes in both may be preferred.

To reduce the significance imposed by the experimenter's instructions on the low-intensity tones used in this study, the tones were not mentioned. The experiment was presented as investigating the correlation of various psychophysiological measures over time, and the earphones were supposedly used to reduce intermittent noises from the elevator servicing the building. A pilot study indicated that this strategem was justified, subjects reporting that they could "still hear some elevator noises." It was, therefore, expected that the significance attributed to the stimuli in this study would be minimal.

Stimuli consisted of $2-\sec 1,000-\mathrm{Hz}$ tones (generated by a modified and calibrated B.W.D. audio oscillator and delivered to earphones) at $20,30,40$, and $50 \mathrm{~dB}$ SPL with a randomized interstimulus interval between 20 and $100 \mathrm{sec}$. Each subject was randomly assigned to a different ordering of these four intensities and stimulation was continued for eight cycles, providing a total of 32 stimuli. The whole recording session lasted $1 \mathrm{~h}$, including preparation time.

\section{RESULTS}

The GSR was defined as a measurable drop in resistance with latency between 1 and $5 \mathrm{sec}$ from stimulus onset. The mean values of GSR magnitude are displayed as a function of intensity in Figure 1 and as a function of repeated cycles in Figure 2. Because the distribution of scores departs markedly from normality [due to the large number of zero scores, a problem not 


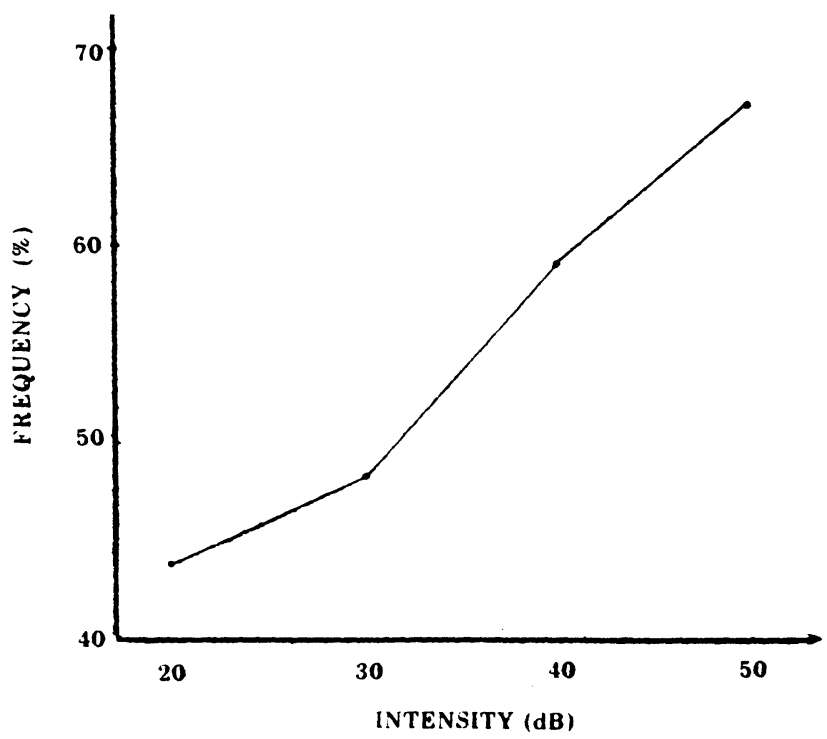

Figure 3. Frequency of occurrence of the GSR as a function of intensity of the stimulus.

considered by Leavy \& Geer (1967)], parametric data analysis could not be used. A Friedman analysis of variance by ranks (Siegel, 1966) carried out on the scores averaged over trials showed the intensity effect to be highly significant $\left(\chi_{\mathrm{r}}^{2}=38.9, \mathrm{df}=3, \mathrm{p}<.001\right)$, as was the trials effect based on scores averaged over intensity $\left(\chi_{\mathrm{r}}^{2}=45.2, \mathrm{df}=7, \mathrm{p}<.001\right)$. The frequency of GSR occurrence was also extracted from the above data and is displayed as a function of intensity in Figure 3 and as a function of repetition of the stimulus complex in Figure 4. These frequencies were subjected to a chi-squared analysis, and both the intensity effect $\left(\chi^{2}=11.8, \mathrm{df}=3, \mathrm{p}<.01\right)$ and the repetition effect $\left(\chi^{2}=24.3, \quad \mathrm{df}=7, \mathrm{p}<.001\right)$ were found to be significant. The frequency data thus support the GSR magnitude data.

\section{DISCUSSION}

The clear habituation effect shown in Figures 2 and 4 supports Sokolov (1963) and is in line with that usually found in GSR work, unlike the lack of habituation reported by Leavy and Geer (1967). This result, together with the significant intensity effect reported here must be partly attributable to the greater within-subjects effect expected from other fields (such as the RT-intensity effect) and partly to the minimal stimulus significance attained in this study. This would be expected to result in a relatively "pure" OR highly dependent on stimulus intensity for the early stimulus presentations and habituating rapidly. The nature of this stimulus intensity effect at low intensities was found, as has been previously reported for higher intensities, to be monotonically increasing with intensity. There is thus no evidence supporting Sokolov's contention of enhanced

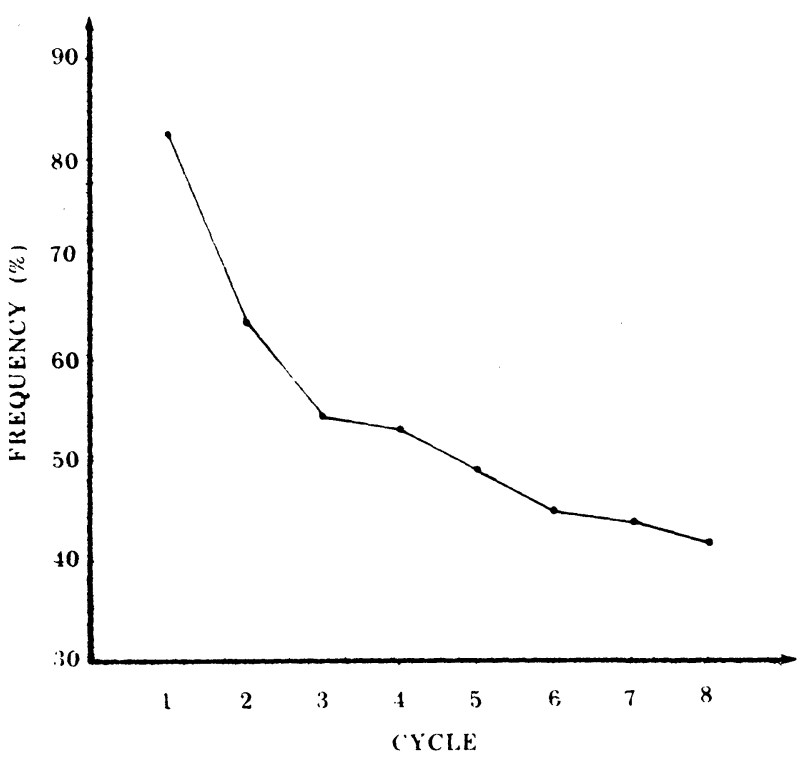

Figure 4. Frequency of occurrence of the GSR as a function of repetition of the stimulus complex.

responding at near threshold intensity levels, and while some allowance must be made for the fact that stimulus presentation was not continued here until complete extinction was obtained (Sokolov's method), there is no evidence to indicate that near threshold stimuli should suddenly become more salient when presented beyond the repetition limit imposed in this study. It would, therefore, appear that such enhancement should be rejected in favor of a linear relationship between GSR magnitude and stimulus intensity over a range of intensity from $120 \mathrm{~dB}$ [established by Raskin et al (1969)] down to $20 \mathrm{~dB}$ (the lower limit used here).

\section{REFERENCES}

Campos, J. J., \& Johnson, H. J. The effects of verbalization instructions and visual attention on $H R$ and SC. Psychophysiology, 1966, 2, 305-310.

Grice, G. R., \& Hunter, J. J. Stimulus intensity effects depend upon the ty pe of experimental design. Psychological Review, 1964, 71, 247-256.

Hovland, C. I., \& Riesen, A. H. Magnitude of galvanic and vasomotor response as a function of stimulus intensity. Journal of General Psychology, 1940, 23, 103-121.

Kohlenberg, $R$. J. Instructions to ignore a stimulus and the GSR. Psychonomic Science, 1970, 19, 220-221.

Leavy, A., \& Geer, J. H. The effect of low levels of stimulus intensity upon the OR. Psychonomic Science, 1967, 9, 105-106.

Lykken, D. T., \& Venables, P. H. Direct measurement of skin conductance: A proposal for standardisation. Psychophysiology, 1971, 8, 656-672.

Montague, J. D., \& Coles, E. M. Mechanism and measurement of the galvanic skin response. Psychological Bulletin, 1966, 65, 261-279.

Raskin, D. C., Kotses, H., \& Bever, J. Autonomic indicators of orienting and defensive reflexes. Journal of Experimental Psychology, 1969, 80, 423-433.

Siegel, S. Nonparametric statistics for the behavioral sciences. New York: McGraw-Hill, 1966.

Sokolov, E. N. Perception and the conditioned reflex. Oxford: Pergamon Press, 1963.

Uno, ${ }^{2}$ \& Grings, W. W. Autonomic components of orienting behavior. Psychophysiology, 1965, 1, 311-321.

(Received for publication June 18, 1974; revision received August 27, 1974.) 